\title{
Externally Resonated Linear Microvibromotor for Microassembly
}

\author{
Kazuhiro Saitou, Dung-An Wang, and Soungjin J. Wou
}

\begin{abstract}
A new method for on-substrate fine positioning of microscale/mesoscale discrete components is presented [1]-[3], where component positions are finely adjusted using microlinear sliders and fixtures on the substrate. Each microlinear slider is actuated by vibratory impacts exerted by two pairs of microcantilever impacters. These microcantilever impacters are selectively resonated by shaking the entire substrate with a piezoelectric vibrator, requiring no need for built-in driving mechanisms such as electrostatic comb actuators, as reported previously [4], [5]. This selective resonance of the microcantilever impacters via an external vibration energy field [6] provides with a very simple means of controlling forward and backward motion of the microlinear slider, facilitating assembly and disassembly of a microcomponent on a substrate. An analytical model of the device is derived in order to obtain, through the simulated annealing algorithm, an optimal design, which maximizes translation speed of the linear slider at desired external input frequencies. Prototypes of the externally resonated linear microvibromotor are fabricated using the three-layer polysilicon surface micromachining process provided by the Microelectronics Center of North Carolina, Research Triangle Park, NC, multiuser microelectromechanical processes service. These prototypes are tested for forward and backward motion via external vibration applied by an piezoelectric flexure vibrator, as well as the horizontal positioning and release of 500- $\mu \mathrm{m}$-square polysilicon chips against a reference fixture element anchored to the substrate. [457]
\end{abstract}

Index Terms-Microactuators, micromechanical resonators, vibrations.

\section{INTRODUCTION}

$\mathbf{T}$ $\mathrm{HE}$ need of microscale/mesoscale assembly of discrete components is becoming evident in complex integrated microsystems that often suffer from low reliability due to the lack of modularity among subsystems. This is especially true for microelectromechanical systems (MEMS), which often require complex electromechanical integration involving the materials incompatible to batch fabrication processes.

Assembly of microdiscrete components is typically done by manual operation of microprobes or microtweezers (e.g., [7]). Such miniaturization of the conventional pick-and-place robotic assembly, however, experiences extreme difficulty in

Manuscript received June 8, 1999; revised December 6, 1999. This work was supported by National Science Foundation under Grant DMI-9813110. Subject Editor, R. T. Howe.

K. Saitou and D.-A. Wang are with the Department of Mechanical Engineering and Applied Mechanics, Tthe University of Michigan at Ann Arbor, Ann Arbor, MI 48109-2125, USA.

S. J. Wou was with the Department of Mechanical Engineering and Applied Mechanics, The University of Michigan at Ann Arbor, Ann Arbor, MI 48109-2125, USA. He is now with Bishop Steering Technology, Sydney, Australia.

Publisher Item Identifier S 1057-7157(00)08021-5. a)
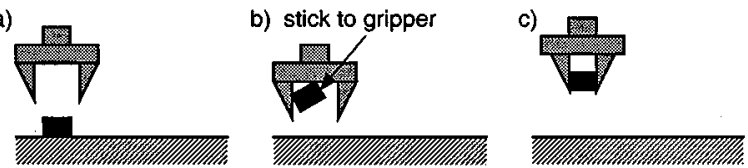

d)
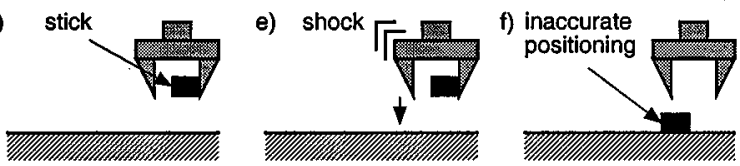

Fig. 1. Typical pick-and-place assembly in microscale (modified from [8]). (a) A gripper approaches to a component. (b) The component sticks to the gripper. (c) The gripper grasps the component. (d) The component is transported to a desired location. (e) The component is released with shock. (f) The component is placed at inaccurate position.

handling and positioning components with sizes less than a millimeter, due to the surface adhesion forces that cause sticking among components and handling devices [8]. Fig. 1 illustrates pick-and-place assembly of a microscale component using a microgripper. Surface adhesion forces such as electrostatic, van der Waals, and surface tension forces cause the component to stick to the gripper during the approach [see Fig. 1(b)] and release [see Fig. 1(d)] phases. Mechanical shock can be applied to the gripper to drop the stuck component [see Fig. 1(e)], with the price of inaccurate positioning of the released component [see Fig. 1(f)].

One way to overcome this problem is to design a device on the substrate that facilitates component positioning so that gross positioning is done in the conventional pick-and-place fashion, ${ }^{1}$ whereas fine positioning is done by an on-substrate positioning device. This concept is illustrated in Fig. 2, where an on-substrate linear actuator pushes an inaccurately positioned microcomponent [e.g., as a result of the "shock release" shown in Fig. 1(e) and (f)] against a fixture anchored to the substrate [see Fig. 2(a)], achieving precise positioning of the component [see Fig. 2(b)]. The linear actuator also should be able to reopen to release the positioned component to facilitate the potential needs for disassembly due to subsystem failure or upgrade [see Fig. 2(c)].

This paper describes a design of such a microlinear actuator for fine positioning of a microscale to mesoscale component on a substrate [1]-[3]. The design is based on a linear microvibromotor reported by Daneman et al. [4], where a microlinear slider is actuated by vibratory impacts exerted by microcantilever impacters driven by electrostatic comb actuators. Dissimilar to

\footnotetext{
${ }^{1}$ Or with other processes that are more effective for gross positioning, e.g., vibratory agitation (see Section II for examples).
} 


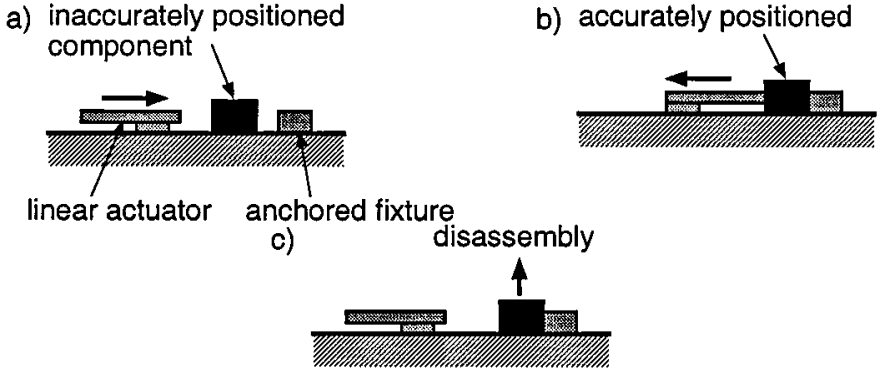

Fig. 2. Precise component positioning and release with a on-substrate linear actuator and a fixture. (a) Inaccurately positioned component. (b) Accurately positioned component. (c) Release of the component for disassembly.

their design, however, these microcantilever impacters are selectively resonated by shaking the entire substrate with a piezoelectric vibrator, requiring no need for built-in driving mechanisms such as electrostatic comb actuators. This selective resonance of the microcantilever impacters via an external vibration energy field [6] provides with a very simple means of controlling forward and backward motion of the microlinear slider, facilitating assembly and disassembly of a microcomponent on a substrate. The double- $\mathrm{V}$ beam suspension design [9] is employed in the microcantilever impacters for larger displacement in the lateral direction while achieving higher stiffness in the transversal direction. An analytical model of the device is derived in order to obtain, through the simulated annealing algorithm, an optimal design, which maximizes translation speed of the linear slider at desired external input frequencies. Prototypes of the externally resonated linear microvibromotor are fabricated using the three-layer polysilicon surface micromachining process provided by the Microelectronics Center of North Carolina (MCNC), Research Triangle Park, NC, multiuser microelectromechancial processes (MUMPS) service.

\section{RELATED WORK}

In the efforts of the development of a bulk assembly method for microscale to mesoscale components, several approaches have been proposed to incorporate self-positioning to microassembly. Yeh and Smith [10] integrated trapezoidal GaAs microblocks on an Si substrate with trapezoidal holes by dispensing these in a carrier fluid (ethanol) onto the $\mathrm{Si}$ substrate. Hadley [11] also developed a similar fluidic self-assembly method for integrating microblocks containing MOS transistors to a glass substrate. Terfort et al. [12], [13] demonstrated that millimeter-scale LED's with operating electrical connections can be self-positioned in water by complementary sculpting the mating surfaces and coating them with hydrophobic materials. Hosokawa et al. [14] experimented with the self-positioning of microparts, which are brought together on a water surface by surface tension of the water. Cohn et al. [15] experimented with the self-positioning of small hexagonal parts $(1 \mathrm{~mm}$ in diameter) by placing a quantity of them on a slightly concave diaphragm that was agitated with a loudspeaker. Böhringer et al. [16] proposed a method to position submillimeter parts using ultrasonic vibration to eliminate friction and adhesion, and electrostatic forces to position and align parts in parallel. While virtually no external positioning/handling of components

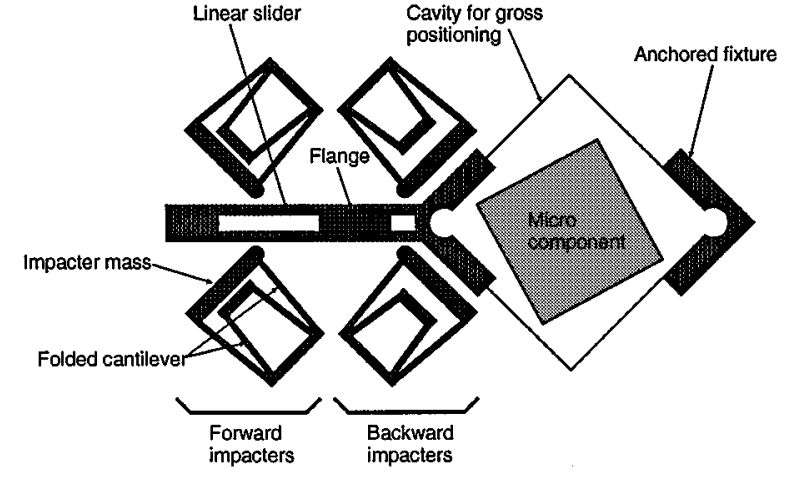

Fig. 3. Schematic top view of the externally resonated linear microvibromotor for on-substrate precise positioning.

is necessary in these methods, the components are often grossly positioned without positive fastening, requiring auxiliary means to achieve precise positioning and fastening. The work presented in this paper would compliment this aspect of the above bulk-positioning methods.

Other work has been done on the use of mechanical force to both self-position and fasten components so that assembly requires less positioning/handling of components. Judy et al. [17] fabricated a laterally deflecting cantilever on the sidewall of a polysilicon mesa, which adjusts the position of other structures attaching to the cantilever, and provides the bearing forces between structures. Burgett et al. [18] used spring-loaded latches to self-position the plates within microfabricated hinges. Prasad et al. [19] fabricated a microsnap fastener with 1-2- $\mu \mathrm{m}$-wide laterally deflecting chamfered latches. These methods do not consider the potential need for disassembly due to subsystem failure or upgrade, hence, nondestructive removal of the fastened components is extremely difficult or even impossible. The reader should also refer to [20] for an excellent review of the current microassembly technologies.

\section{DESIGN}

\section{A. Operational Principle}

Our design of the externally resonated linear microvibromotor for microassembly is based on a linear microvibromotor reported by Daneman et al. [4], where a microlinear slider is actuated by vibratory impacts exerted by microcantilever impacters. Dissimilar to their design, however, these microcantilever impacters are selectively resonated by external piezoelectric vibration, requiring no need for built-in driving mechanisms such as electrostatic comb actuators.

As illustrated in Fig. 3, it consists of a linear slider located between two pairs of folded cantilever impacters anchored on the substrate, which can exert forward and backward vibratory impacts to the sides of the slider, depending on which pair of impacters is resonated by external vibration. Fig. 4 illustrates the three-step operation of the linear microvibromotor. First, the substrate is shaken with a piezoelectric vibrator at the frequency $f_{1}$. This external vibration resonates only the forward impacters, causing the linear slider to move to the right-hand side [see Fig. 4(a)]. This motion causes the slider to push a microcomponent against an anchored fixture, achieving precise positioning 


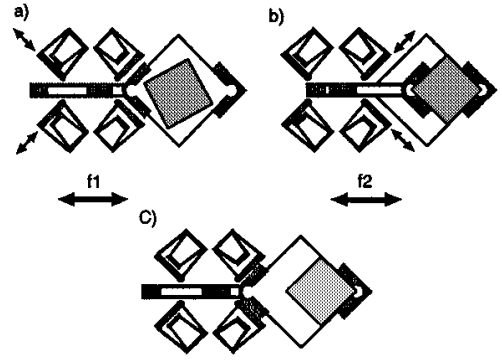

Fig. 4. Three-step operation of the externally resonated linear microvibromotor. (a) The resonance of forward impacters. (b) The resulting forward sliding motion and the resonance of the backward impacters. (c) The resulting backward sliding motion.

[see Fig. 4(b)]. Next, the substrate is shaken at the frequency $f_{2}$. This external vibration resonates only the backward impacters and moves the slider to the left-hand side [see Fig. 4(c)], releasing the positioned component.

This selective resonance of the microcantilever impacters via an external vibration energy field [6] provides with very simple means of controlling forward and backward motion of the microlinear slider, without explicit routing to direct energy to each of the impacters. This property of the selective resonance would be particularly useful in the situation where a number of linear microvibromotors are implemented in a two-dimensional array in order to position multiple microcomponents simultaneously. By designing the forward and backward impacters to have different resonance frequencies, each linear microvibromotor in the array can be operated independently by the external piezoelectric vibrations driven by the sum of the signals with appropriate resonance frequencies [6].

In Fig. 4, note that the direction of the external vibration is not parallel to the direction of impacters' oscillation (i.e., the direction of impact). Therefore, it is the component of the external vibration parallel to the direction of impact that causes the resonance in the microimpacters. Another component of external vibration causes the impacters to deform perpendicular to the direction of impact, which is undesirable for efficient operation of the linear microvibromotor. The microcantilever impacters, therefore, should have high stiffness in the direction perpendicular to the direction of impact, while keeping the relatively low stiffness in the direction of impact. To achieve this goal, the double- $\mathrm{V}$ beam suspension design, shown in Fig. 5(b), is employed in the microcantilever impacters, which realizes higher transversal stiffness than the conventional folded parallel beam design without affecting the lateral stiffness [9].

\section{B. Modeling}

Equations of motions of a lumped parameter model of the impacter-slider system, illustrated in Fig. 3, is derived in order to obtain an optimal design, which maximizes translation speed of the linear slider at desired external input frequencies. The modeling presented below follows the basic procedure described by Lee and Pisano [21] for their micromechanical impact test structures, and by Daneman et al. [4] for their electrostatic linear vibromotor.

Fig. 6 shows the close-up view of an impacter mass and the slider sidewall, where $(x, y)$ denote the coordinate system for a)

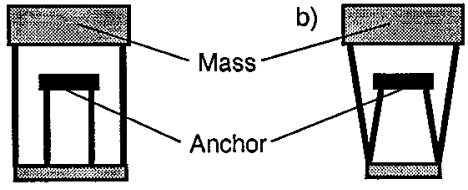

Fig. 5. Microcantilever designs. (a)The conventional folded-beam. (b) Double-V beam.

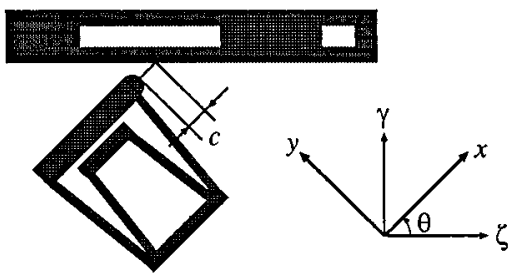

Fig. 6. Close-up view of an impacter mass and the slider side wall (modified from [4]).

the impacter position, and $(\zeta, \gamma)$ denote the coordinate system for the slider position. The $x-y$-axes are rotated from $\zeta-\gamma$-axes by the impact angle $\theta$. The following assumptions are made in derivation of the lumped parameter model [4].

- The motion of impacters and the slider in the direction perpendicular to the substrate is minimal and does not affect their in-plane motion. ${ }^{2}$

- The impacters are completely rigid in the $y$-direction.

- The slider is completely rigid and there is no clearance in the $\gamma$-direction between the flange and the slider.

- There is no friction between the substrate and impacter mass.

- An impact between the impacter mass and slider sidewall occurs instantaneously.

- Impacts by the two impacters in a pair occur simultaneously.

Given these assumptions, an impacter can be modeled as a simple mass-spring-damper system with an external force input $f_{\text {ext }}(t)$

$$
m \ddot{x}+b \dot{x}+k x=f_{\text {ext }}(t)
$$

where $m, b$, and $k$ are the mass, viscous damping coefficient, and spring constant of an impacter, respectively. Assuming Coutette air flow between the substrate and impacter mass, and small lateral displacement of the folded beams, these parameters are expressed as [22], [9]

$$
\begin{aligned}
m & =\rho A t+2 \rho h w l \\
b & =\mu \frac{A}{d} \\
k & =E h\left(\frac{w}{l}\right)^{3} \frac{4}{\cos \phi}\left(\frac{2}{\cos \phi}+1\right)
\end{aligned}
$$

where $\rho$ is the mass density of the impacter material (polysilicon), $A$ and $t$ are the planer area (including the area of the joining member of two folded beams) and thickness of the impacter mass, respectively, $h, w$, and $l$ are the height, width, and

${ }^{2}$ This can be justified by the fact the vertical displacement of the piezoelectric flexure vibrator used to shake the substrate (described in Section IV-B) is $0.6-3 \%$ of its horizontal displacement. 
total length of the two segments of a V-beam, respectively, $\mu$ is the viscosity of the fluid in the environment (e.g., air), $d$ is the vertical gap between the substrate and impacter mass, $E$ is Young's modulus of the beam material (polysilicon), and $\phi$ is the half of the angle between the two segments of a V-beam. Assuming the substrate is shaken with the external vibration $\zeta=Z_{0} \cos (\omega t)$ in the $\zeta$-direction, the inertial force $f_{\text {ext }}(t)$ exerted to a impacter is

$$
f_{\mathrm{ext}}(t)=m \omega^{2} Z_{0} \cos \theta \cos (\omega t)
$$

Similarly, the equation of motion of the linear slider is given as

$$
M \ddot{\zeta}=F(t)
$$

where $M$ is the mass of the slider and $F$ is a net force exerted to the slider

$$
F(t)=\left\{\begin{array}{c}
0, \quad \text { if } \dot{\zeta}=0 \text { and }\left|F_{\text {ext }}(t)\right|<F_{s} \\
-B \dot{\zeta}+F_{\text {ext }}(t)-F_{d} \dot{\zeta} /|\dot{\zeta}| \quad \text { otherwise }
\end{array}\right.
$$

where $B$ is the viscous damping coefficient of the slider, $F_{\text {ext }}(t)=M \omega^{2} Z_{0} \cos (\omega t)$ is the inertial force exerted to the slider, and $F_{s}$ and $F_{d}$ are static and dynamic frictional forces, respectively. The parameters $M$ and $B$ are given similarly to (2) and (3).

An oblique impact of the impacter tips to the slider sidewall is modeled as an impact with restitution in the $\gamma$-direction, and an impact with instantaneous momentum transfer in $\zeta$-direction [21]. Let $c$ be the distance between the impacter tip and the slider sidewall measured in the $x$-direction, as shown in Fig. 6 . If $x<c$, there is no impact. At $x=c$, the impacter tip contacts the slider sidewall. In the $\gamma$-direction, the following boundary condition models the energy dissipation of the impacter at an impact:

$$
v^{+} \sin \theta=-e v^{-} \sin \theta
$$

where $v^{-}$and $v^{+}$are impacter velocities in the $x$-direction right before and right after the impact, and $e$ is the coefficient of restitution. In the $\zeta$-direction, linear momentum is transferred from the impacters to the slider. Considering there are two impacters to drive the slider

$$
2 m v^{+} \cos \theta+M V^{+}=2 m v^{-} \cos \theta+M V^{-}
$$

where $V^{-}$and $V^{+}$are slider velocities in the $\zeta$-direction right before and right after the impact. Rearranging (8) and (9) gives the boundary condition to model the energy transfer to the slider at an impact

$$
V^{+}=V^{-}+2 \frac{m}{M}(1+e) v^{-} \cos \theta
$$

The equations of motion defined as (1)-(10) are numerically integrated with the fourth-order Runge-Kutta method to predict
TABLE I

Physical Constant VALUES UsEd IN THE Simulation

\begin{tabular}{c|c|c}
\hline param [unit] & value & note \\
\hline \hline$\rho\left[\mathrm{g} / \mathrm{cm}^{3}\right]$ & 2.33 & LPCVD poly-Si [22] \\
\hline$\mu[\mathrm{mPa} \cdot \mathrm{s}]$ & 0.0 & $10 \mathrm{~m}$ Torr sir \\
\hline$E[\mathrm{GPa}]$ & 169 & poly-Si [23] \\
\hline$F_{s}[\mu N]$ & 20 & between LPCVD poly-Si layers [4] \\
\hline$F_{d}[\mu N]$ & 5 & between LPCVD poly-Si layers [4] \\
\hline$e$ & 0.5 & between LPCVD poly-Si side walls [5]
\end{tabular}
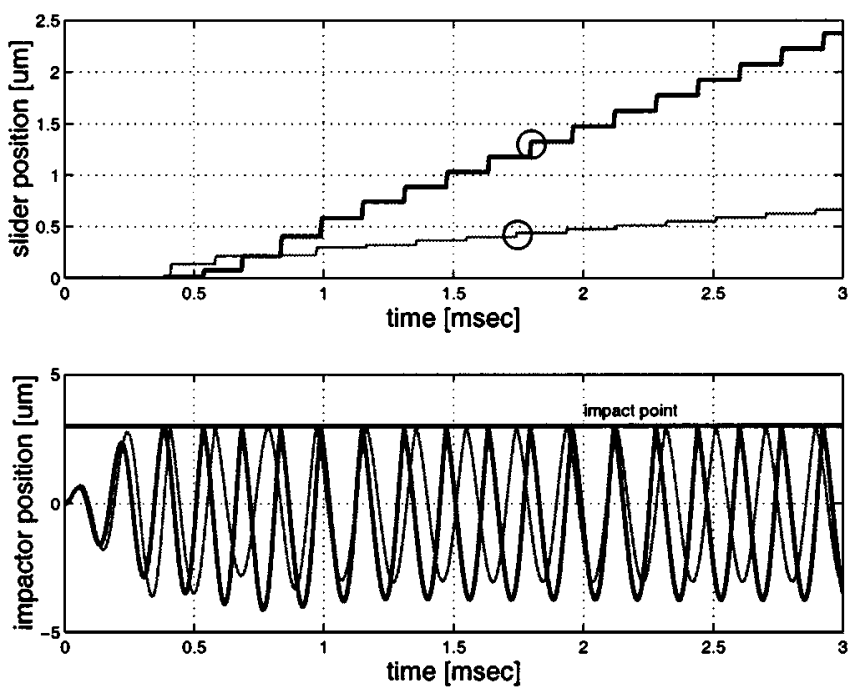

Fig. 7. Example of the simulated vibromotor performances with $\nu=\omega / 2 \pi=$ $5.2 \mathrm{kHz}$ (thin line) and $\nu=\omega / 2 \pi=6.2 \mathrm{kHz}$ (thick line). The top figure shows the time plot of the slider position and the bottom figure shows the time plot of the impacter position. Close-up views of the circled regions in the top figure are shown in Fig. 8.

and optimize a design of the externally resonated linear microvibromotor. The values of the $\phi$ and $\theta$ used in the numerical simulation are $15^{\circ}$ and $45^{\circ}$, respectively. The values of $d, h$, and $t$ are constrained by the MUMP's process provided by MCNC used for device fabrication discussed in Section IV. They are set to be $0.75,2.0$, and $3.5 \mu \mathrm{m}$, respectively. The physical constant values used in the simulation are shown in Table I. Note that viscosity of the environmental fluid $\mu$ is set to zero since the device will be tested in very low pressure (10 mtorr) in Section IV-B. The values of $F_{s}$ and $F_{d}$ account for not only the friction between the substrate and the slider, but also the slop between the slider and its guide, and are estimated based on [4] since the slider size and its fabrication process are virtually identical.

Fig. 7 shows the results of numerical integration of the above equations of motion (1)-(10) in the time period from $t_{0}=$ $0.0 \mathrm{~ms}$ to $t_{f}=3.0 \mathrm{~ms}$ with two external input frequencies: $\nu=\omega / 2 \pi=5.2 \mathrm{kHz}$ (thin line) and $\nu=\omega / 2 \pi=6.2 \mathrm{kHz}$ (thick line). For each input frequency, the top figure shows the time plot of the slider position, and the bottom figure shows the time plot of the impacter position. In order to facilitate a fair comparison of device performances with different input frequencies, the power input from the external vibration is kept constant. Since the power input from the external vibration is proportional to $Z_{0}^{2} \cdot \omega^{3}$, this quantity is kept at a constant value 


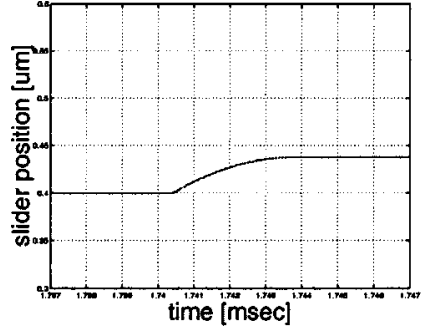

(a)

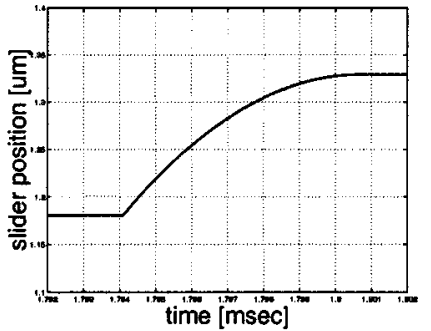

(b)
Fig. 8. Close-up views of one "step" indicated with circles in the top figure of Fig. 7.

of $50 \mathrm{~m}^{2} / \mathrm{s}^{3}{ }^{3}$ The parameter values common to both figures are $A=1.5 \times 10^{4} \mu \mathrm{m}^{2}, w=4.0 \mu \mathrm{m}, l=1242 \mu \mathrm{m}, c=3.0 \mu \mathrm{m}$, and the slider area is $8.0 \times 10^{4} \mu \mathrm{m}$. These values give the impacter (undamped) natural frequency $\nu_{n}=\omega_{n} / 2 \pi=5.2 \mathrm{kHz}$, where $\omega_{n}=\sqrt{k / m}$. The initial state $\left(x\left(t_{0}\right), \dot{x}\left(t_{0}\right)\right)$ is $(0.0,0.0)$ in both cases.

The step-like motion of the slider position is a result of the almost instantaneous acceleration due to the impact followed by rapid deceleration due to the transition from dynamic to static friction. The close-up views of one "step" indicated by the circled regions in the top figure are shown in Fig. 8(a) for $\nu=$ $\omega / 2 \pi=5.2 \mathrm{kHz}$ (thin line) and in Fig. 8(b) for $\nu=\omega / 2 \pi=$ $6.2 \mathrm{kHz}$ (thick line), respectively. The impacter position exhibits a saw-teeth-like asymmetric waveform and the steady-state amplitude is asymmetric with respect to its neutral position, due to the "cut off" of oscillation caused by collisions to the slider sidewall. This is more evident in the case of $\nu=\omega / 2 \pi=6.2 \mathrm{kHz}$ (thick line) due to the earlier "cut off" of oscillation.

It should be noted that when the impacter is driven with its natural frequency $\nu_{n}=5.2 \mathrm{kHz}$ (thin line in Fig. 7), the oscillation amplitude does not become large enough to provide the impact at each cycle, resulting in uneven "steps" in the slider motion. At higher frequency $6.2 \mathrm{kHz}$ (thick line in Fig. 7), on the other hand, the impacter rapidly acquire enough momentum to impact the wall at each cycle. As a result, an average slider velocity with $6.2 \mathrm{kHz}$ is approximately four times larger than with $5.2 \mathrm{kHz}$. This increase in the system resonance frequency is due to the nonlinear "hardening spring" behavior observed in many oscillatory systems involving impacts [24]-[27], most notably in a micromechanical vibroimpact system [21]. Such systems are often approximated by a damped Duffing oscillator [28]

$$
\ddot{x}+2 \alpha \dot{x}+\beta x+\epsilon x^{3}=f(t)
$$

where $\alpha, \beta, \epsilon>0$ are constants and $f(t)$ is a periodic function of time $t$.

As many other nonlinear oscillatory systems, the Duffing-like nonlinear systems exhibit instabilities where a small perturbation of the initial state $\left(x\left(t_{0}\right), \dot{x}\left(t_{0}\right)\right)$ completely changes the frequency response of the system [24]-[27]. Such instabilities can, in fact, occur in the impacter-slider system, as defined in (1)-(10) since the initial state of the impacter mass may vary at

\footnotetext{
${ }^{3}$ For instance, this value gives the external vibration amplitude $Z_{0}=1.27 \mu \mathrm{m}$ at the frequency $\nu=\omega / 2 \pi=5.0 \mathrm{kHz}$, which is reasonable for actuation with a piezoelectric vibrator.
}

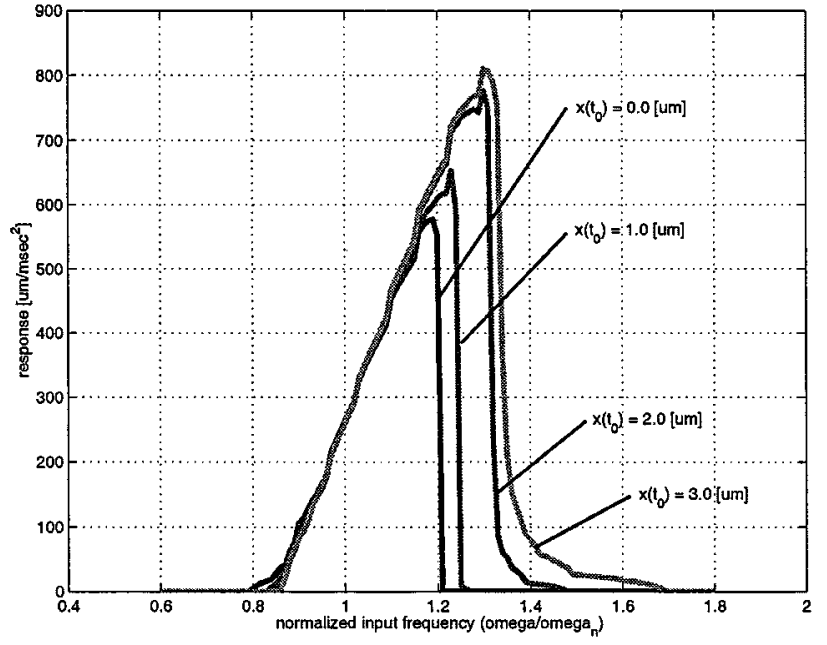

Fig. 9. Frequency responses of the impacter-slider system for the initial impacter positions $x\left(t_{0}\right)=0.0,1.0,2.0,3.0 \mu \mathrm{m}$.

every operation of the device due to the initial adjustments in external input frequency and amplitudes. Fig. 9 shows the frequency responses of the impacter-slider system with the same parameter as in Fig. 7 with the initial impacter positions $x\left(t_{0}\right)=$ $0.01 .0,2.0,3.0 \mu \mathrm{m}$. Based on the observation that the change in the slider position at an impact is a monotonously increasing function of the linear momentum of the impacters right before the impact, the system response is defined as follows:

$$
r\left(\mathbf{x}_{0}, \mathbf{p}, \nu\right)=\frac{m \sum_{k=2}^{n} v^{-}\left(t_{k}\right)}{t_{f}-t_{0}}
$$

where $\mathbf{x}_{0}=\left(x\left(t_{0}\right), v\left(t_{0}\right)\right)$ is the initial state, $\mathbf{p}$ is a vector of the system parameters, $\nu$ is the input frequency, $m$ is the mass of an impacter given by (1), $n$ is the number of impacts occurred during the time period from $t_{0}$ to $t_{f}$, and $t_{k}, k=2,3, \ldots, n$ is the time when the second impact, the third impact, etc. occurred. Note that $v^{-}\left(t_{1}\right)$ is not included in the above sum to avoid accounting for the first impact due to the initial impacter position. As shown in Fig. 9, the input frequencies at which the sudden transitions in the system response occur (bifurcation points [28]) varies for different initial impacter positions. It is desirable, therefore, to drive the device in the frequency where such instability does not occur even in the presence of small perturbation in the initial impacter state.

Although the above dynamic model shares many similarities to the one presented in [4], there are two essential differences to be noted. First, the model in [4] was solved by piercing together the independently solved analytical solutions for impact and nonimpact cases, whereas the above solution is obtained through numerical integration of the system model. In [4], piercing together two analytical solutions was feasible since the impacter neutral position could be adjusted with the dc bias to the comb actuators such that the impact to the slider sidewall occurs just at the free oscillation amplitude of the impacters, minimizing the nonlinear effects due to the impact. On the other hand, the system model needs to be numerically solved in the above since our interest is the full dynamic behavior of the 
system in "early" impact cases, where the impacts occur far before the impacters reach their free oscillation amplitudes. In such cases, piercing together two analytical solutions cannot predict the dynamic behavior of the system, most notably the nonlinear effects illustrated in Fig. 9.

Second, in [4], the slider sidewall was modeled as a very stiff spring and a damper, whereas in the above, it is modeled as a rigid wall with restitution. Modeling the sidewall as a stiff spring and a damper provides a straightforward analytical solution during impact [24], [25], [4], although numerical integration of such a model requires a very small time step during impact, resulting in increased computational time. On the other hand, the restitution model, employed in numerous work on impact dynamics modeling (e.g., [5], [21], [26], [27]) requires much less computational time for numerical integration due to the assumption of the instantaneous impact. The simple restitution model is employed in the above since, in our work, numerically solving the system model is essential, and also the numerical simulation is repeatedly used during design optimization discusses in the following section.

\section{Design Optimization}

The system frequency response, as defined in (12), provides an objective function for an optimal vibromotor design that maximizes translation speed of the linear slider at a desired external input frequency. For reliable operation of the device, the design should be optimized for maximum slider speed in the presence of small perturbation of the initial state. The instability of the system response illustrated in Fig. 9 requires the optimization to maximize the system response at the worst case scenario, i.e., to maximize the minimum response among possible perturbation of the initial condition. In addition, the forward impacters should not respond to the input frequency for the backward impacters, and vise versa. These considerations suggest the following max-min formulation of an optimal design problem of the forward impacters:

$$
\begin{array}{ll}
\max _{\mathbf{p}} & \min _{\mathbf{x}_{0}} r\left(\mathbf{x}_{0}, \mathbf{p}, \nu_{f}\right) \\
\text { s.t. } & \max _{\mathbf{x}_{0}} r\left(\mathbf{x}_{0}, \mathbf{p}, \nu_{b}\right)=0 \\
& \mathbf{x}_{0} \in D \\
& \mathbf{p} \in P
\end{array}
$$

where $r$ is the system frequency response, as defined in (12), and $\nu_{f}$ and $\nu_{b}$ are the input frequencies for the forward and backward impacters, respectively. Equation (13) constraints that a feasible design should not respond to the backward input frequency $\nu_{b}$ regardless of the initial condition $\mathbf{x}_{0}$. Switching $\nu_{f}$ in (13) and $\nu_{b}$ in (14) gives a formulation for the backward impacters.

Note that the evaluation of $r\left(\mathbf{x}_{0}, \mathbf{p}, \nu\right)$ requires only the impacter dynamics, as defined in (1)-(5), and (8). The design parameters $\mathrm{p}$, therefore, consists only of the ones for the impacters: the planer area $A$ of the impacter mass, the width $w$ and the total length $l$ of the two segments of a V-beam, and the distance $c$ between the impacter tip and the slider sidewall measured in the $x$-direction. The lower bounds of these parameters are given by the impacter geometry illustrated in Fig. 6 and the minimum feature length $2.0 \mu \mathrm{m}$, as specified

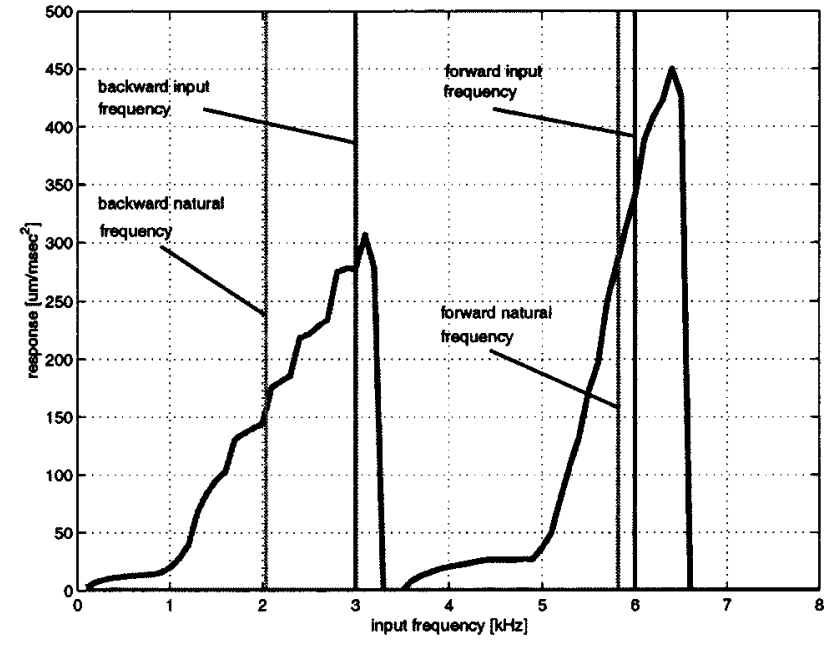

Fig. 10. Frequency responses of the forward (right-hand side) and backward (left-hand side) impacters from an example optimization for $\nu_{f}=6.0 \mathrm{kHz}$ and $\nu_{b}=3.0 \mathrm{kHz}$

TABLE II

RESULT FROM AN EXAMPLE OPTIMIZATION FOR $\nu_{f}=6.0 \mathrm{kHz}$ AND $\nu_{b}=3.0 \mathrm{kHz}$

\begin{tabular}{c|c|c}
\hline param & forward & backward \\
\hline \hline$A\left[\mu m^{2}\right]$ & 13544 & 45446 \\
\hline$w[\mu m]$ & 5.6774 & 4.0354 \\
\hline$l[\mu m]$ & 795.74 & 795.27 \\
\hline$c[\mu m]$ & 5.2046 & 2.6676 \\
\hline$\nu_{n}[k H z]$ & 5.8199 & 2.0385 \\
\hline$x\left(t_{0}\right)[\mu m]$ & -2.6000 & -2.6676 \\
\hline
\end{tabular}

by the MUMPS process. Since these parameters are not upper bounded, the set $P$ is defined as follows:

$$
\begin{aligned}
& P=\{(A, w, l, c) \mid 20.0 \leq A<\infty, 2.0 \leq w<\infty \\
&8.0 \leq l<\infty, 2.0 \leq c<\infty\} .
\end{aligned}
$$

It is assumed that the perturbation in the initial condition is only in the initial impacter position and is bounded by $-c$ and $c$. In other words,

$$
D=\left\{\left(x\left(t_{0}\right), v\left(t_{0}\right)\right) \mid-c \leq x\left(t_{0}\right) \leq c, v\left(t_{0}\right)=0\right\} .
$$

Using $r\left(\mathrm{x}_{0}, \mathrm{p}, \nu\right)$ as an objective function rather than more direct measures of the slider speed, e.g., $\zeta\left(t_{f}\right)$, has two practical advantages for design optimization. First, the evaluation of $r$ is far less computationally expensive than the evaluation of the quantities involving the slider dynamics such as $\zeta\left(t_{f}\right)$. Second, the prediction of the device performance based on $r$ is not necessarily less accurate than the prediction based on the slider dynamics since it does not involve phenomenological constants such as $F_{s}$ and $F_{d}$, whose accurate estimates are extremely difficult to obtain during design stage. ${ }^{4}$

Since the gradient-based nonlinear programming algorithms [29] fail due to the discontinuous change in the system response, illustrated in Fig. 9, the above optimization problem is solved

\footnotetext{
${ }^{4}$ Section IV discusses the effects of the static and dynamic frictional forces to the slider motion, and the estimation of these values based on the results from the operation experiments of the fabricated devices.
} 
using the simulated annealing algorithm [30], combined with sequential quadratic programming (SQP) for local improvements. Table II shows a result from an example optimization ${ }^{5}$ of the forward and backward impacters for the forward input frequency $\nu_{f}=6.0 \mathrm{kHz}$ and the backward input frequency $\nu_{b}=3.0 \mathrm{kHz}$. Note that the initial impacter position $x\left(t_{0}\right)$ of the forward impacter that gives minimum response is approximately $-0.5 c$, not the minimum possible value $-c$ as for the backward impacter. This contradicts the trend illustrated in Fig. 9, where the system response becomes smaller as $x\left(t_{0}\right)$ decreases. Further analyses reveal that for $-c \leq x\left(t_{0}\right)<-0.5 c$, the first impact due to the large initial deflection triggers bifurcation in the response, which results in the response larger than for $x\left(t_{0}\right)=-0.5 c$.

Fig. 10 shows the frequency responses of the forward (right-hand side) and backward (left-hand side) impacters in Table II. Also plotted in this figure are the forward and backward input frequencies, and the undamped natural frequencies of the optimal impacters. It can be easily seen from the figure that the shapes and the relative location of the two response curves are optimized such that the forward impacter has a maximum response at the forward input frequency while achieving zero response at the backward input frequency and vise versa.

\section{FABRICATION AND TESTING}

\section{A. Fabrication}

A $5 \times 5$ array of prototypes of externally resonated linear microvibromotors is fabricated using the three-layer polysilicon surface micromachining process provided by the MCNC MUMPS service, where the bottom polysilicon layer serves as a ground plane, and the middle and the top polysilicon layers are used for micromechanical structures. Fig. 11 illustrates a basic flow of the MUMPS process. ${ }^{6}$ A series of figures shows transversal cross sections of the microlinear slider being fabricated. First, the bottom polysilicon layer (referred to as Poly0) is deposited and patterned on a silicon substrate using low-pressure chemical vapor deposition (LPCVD), as shown in Fig. 11(a). This is followed by the deposition and patterning of a $0.75-\mu \mathrm{m}$-thick sacrificial layer of LPCVD phosphosilicate glass (PSG). Dimples are wet etched on this PSG layer to reduce friction between the bottom and middle polysilicon layers at the completion of the fabrication process [see Fig. 11(b)]. On top of the PSG layer, a 2.0- $\mu$ m-thick LPCVD polysilicon layer (referred to as Poly1) is deposited and patterned. Fig. 11(c) shows the cross-sectional pattern of the slider made of Poly1. After the deposition and patterning of another PSG sacrificial layer [shown in Fig. 11(d)], and a $1.5-\mu \mathrm{m}$ polysilicon layer [referred to as Poly2, shown in Fig. 11(e)], the PSG layers are dissolved in an etching solution (HF), releasing the mechanical structure made of Poly1 and Poly2 [see Fig. 11(f)].

Figs. 12 and 13 show an SEM of an array of the fabricated devices with "dummy" microcomponents, and a close-up view of one device, respectively. Each vibromotors in the array is

\footnotetext{
${ }^{5}$ In this particular example, $\mu=0.0179 \mathrm{mPa} \cdot \mathrm{s}$ is used assuming the operation in the air at a room temperature.

${ }^{6}[$ Online.] Available: http://mems.mcnc.org/mumps.html
}
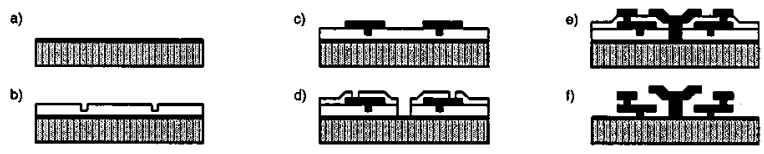

Fig. 11. Basic flow of the MUMPS process (modified from [4]). (a) Deposit and pattern the bottom polysilicon layer. (b) Deposit and pattern the first PSG sacrificial layer. (c) Deposit and pattern the middle polysilicon layer. (d) Deposit and pattern the second PSG sacrificial layer. (e) Deposit and pattern the top polysilicon layer. (f) Dissolve the sacrificial layers in HF solution.

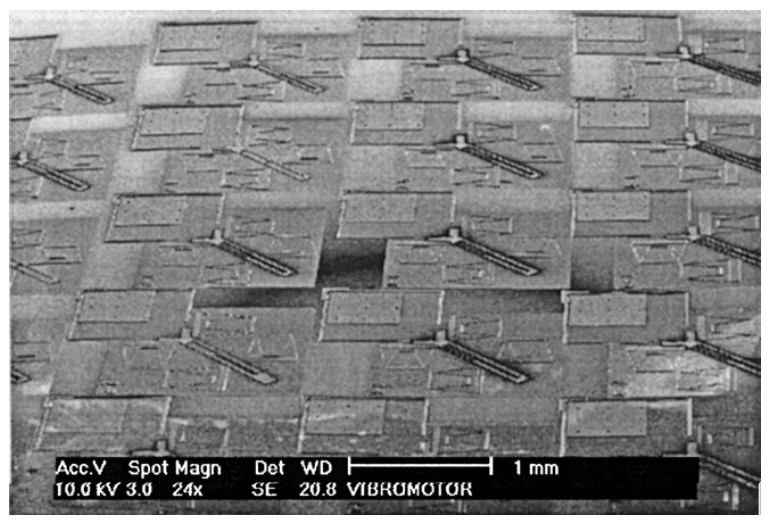

Fig. 12. SEM of an array of the externally resonated microvibromotors with "dummy" microcomponents of $500 \mu \mathrm{m} \times 500 \mu \mathrm{m}$.

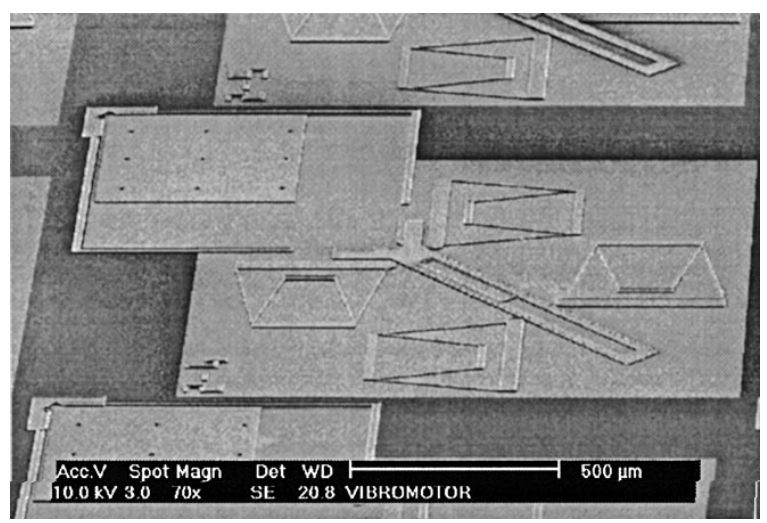

Fig. 13. SEM of a close-up view of the externally resonated microvibromotor.

designed for a different driving frequency to allow potentially selective activation via an external piezoelectric vibrator. The size of the dummy square components is $500 \mu \mathrm{m} \times 500 \mu \mathrm{m}$, made with a Poly1 layer in the MUMPS process. These dummy microcomponents are anchored to the substrate with a very thin polysilicon structure, which is supposed to be broken with a probe tip at testing.

\section{B. Testing}

The fabricated devices are tested using the experimental apparatus shown in Fig. 14, where the die containing the device array is attached to a piezoelectric flexure vibrator that provides a horizontal vibration to drive the microlinear vibromotors. The die and piezoelectric vibrator is placed in a steel vacuum chamber capable of producing gauge pressure of $10 \mathrm{mtorr}$. The top of the chamber is covered with a 0.5 -in-thick transparent Lexan plate with an O-ring to allow observation of the device motion through the microscope. A piezoelectric flexure element 


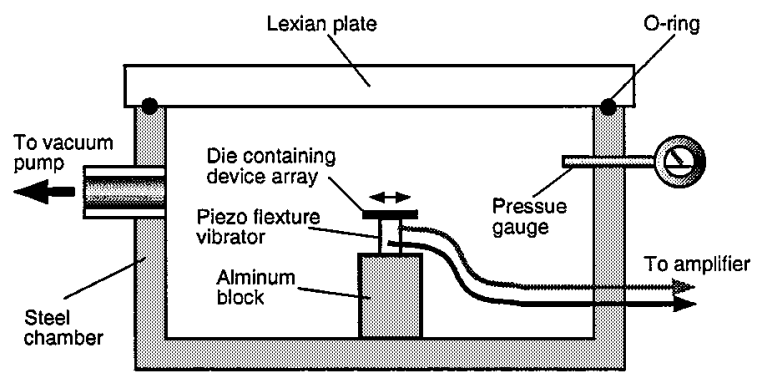

Fig. 14. Experimental apparatus for shaking experiments.

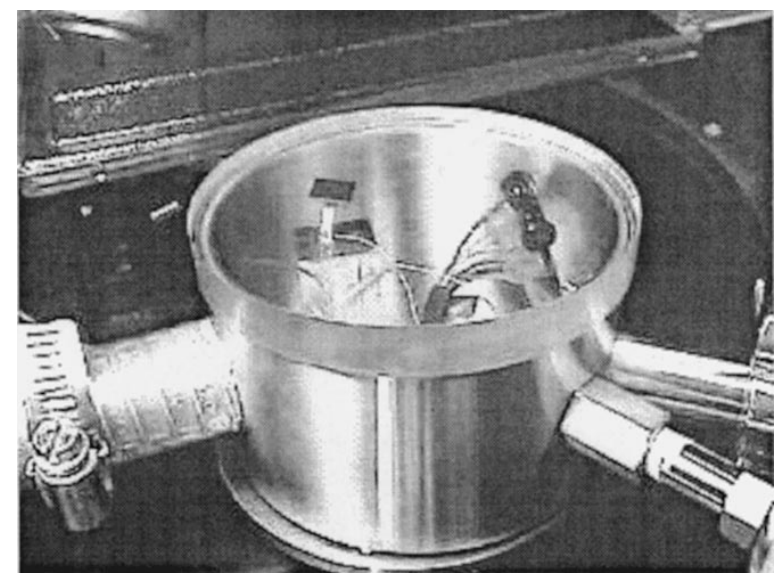

Fig. 15. Overview of experimental setup for shaking experiments.

with static displacement $0.5 \mathrm{~mm} / \mathrm{V}$ and capacitance $1 \mathrm{nF}$ from Sensor Technology, Ltd., Collingwood, Ont., Canada, is used as a vibrator, which is driven by a fixed-gain $(\times 20)$ linear amplifier with peak output power 40-W from Piezo Systems Inc., Cambridge, MA. The input sinusoidal signal to the amplifier is supplied by a Hewlett-Packard 33210A function generator. Fig. 15 is $\mathrm{s}$ a photograph of the experimental apparatus placed under microscope.

Using the experimental setup, the control of forward and backward slider motion is successfully realized by the selective resonance of forward and backward impacters. Figs. 16 and 17 show sequences of snapshots from shaking experiments in atmospheric pressure for forward and backward motions, respectively. For clarification, solid lines are drawn along the edges of the slider and chip in each snapshot. As seen in Fig. 16, a dummy chip is successfully positioned against a reference fixture via the push of the slider realized by its forward motion. The subsequent release of the positioned chip is also realized by the backward slider motion, as shown in Fig. 17. During the experiments, it is observed that once shaking starts the dummy chip keeps oscillating around without sticking to the substrate, and is very easily pushed by the slider. Due to this oscillation, however, the chips sometimes jump out of the square area surrounded by the Poly1 and Poly2 walls (see Fig. 13) that are only $2-\mu \mathrm{m}$ high, and become impossible to be positioned.

The forward and backward slider velocities of a selected device in the fabricated array are measured using the digital video images taken during the shaking experiments. In order to investigate the effect of acoustic streaming forces of the air near the surface of the substrate, the experiments are conducted both
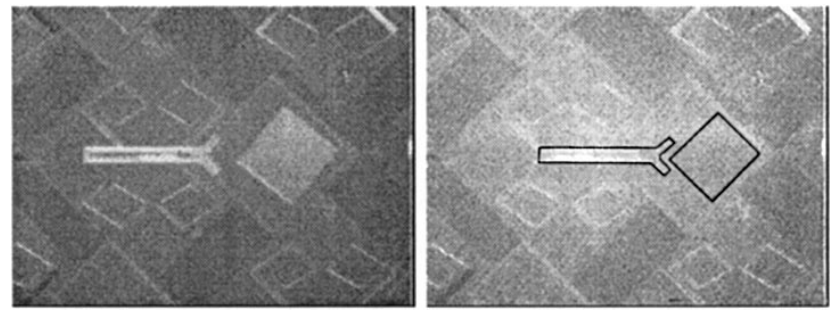

Fig. 16. Sequence of forward motion of a prototype vibromotor: before (left-hand side), and after (right-hand side) the resonance of forward impacters. For clarification, solid lines are drawn along the edges of the slider and chip.
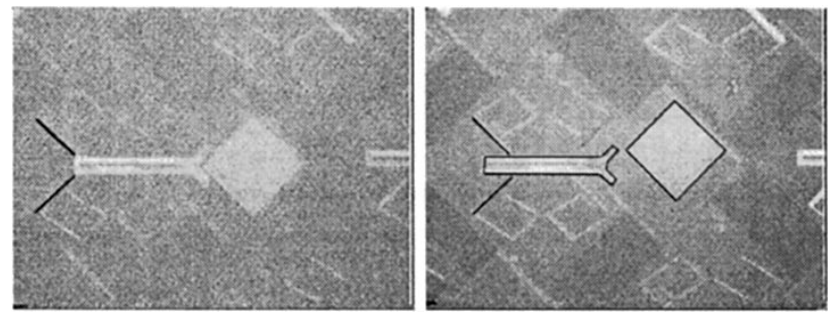

Fig. 17. Sequence of backward motion of a prototype vibromotor: before (left-hand side), and after (right-hand side) the resonance of backward impacters. For clarification, solid lines are drawn along the edges of the slider and chip.

TABLE III

Dimensions of the DeVICE USED For Velocity MEASUREMENTS

\begin{tabular}{c|c|c}
\hline param & forward & backward \\
\hline \hline$A\left[\mu m^{2}\right]$ & 15428 & 15428 \\
\hline$t[\mu \mathrm{m}]$ & 2.0 (Poly1 only) & 3.5 (Poly1 and Poly2) \\
\hline$w[\mu \mathrm{m}]$ & 3 & 3 \\
\hline$l[\mu \mathrm{m}]$ & 555 & 555 \\
\hline$c[\mu \mathrm{m}]$ & 2 & 2 \\
\hline
\end{tabular}

in low pressure (10 mtorr) and in normal atmosphere pressure. The velocities are measured as the average translation distance of the slider edges in the video images between the two consecutive frames. Table III shows the dimensions of the device used for the velocity measurements. The input signal of $10-\mathrm{V}$ sinusoidal wave with no dc offset is amplified to $69.4-\mathrm{V}$ rms to drive the piezoelectric vibrator at frequencies $\nu_{f}=6550 \mathrm{~Hz}$ for forward impacters and $\nu_{b}=7650 \mathrm{~Hz}$ for backward impacters. During the testing, the input signals are turned on instantaneously without ramp up nor frequency sweep. The amplitudes of both horizontal and vertical vibration are measured using Philtec D63 fiber-optic displacement sensor. The horizontal vibration amplitudes at $\nu_{f}=6550 \mathrm{~Hz}$ and $\nu_{b}=7650 \mathrm{~Hz}$ are 2.80 and $0.55 \mu \mathrm{m}$, respectively. The measured vertical vibration amplitudes at both frequencies are $0.02 \mu \mathrm{m}$, hence, the motion of the substrate is in virtually horizontal direction only.

Figs. 18 and 19 show the 34 forward velocity measurements and the 34 backward velocity measurements in 10-mtorr pressure, respectively. No dummy chips are pushed during these measurements. Discrete "jumps" in the measured velocities are due to discrete time associated with the frame speed of the video equipment. The averages of the all measured velocities are 4024 $\mu \mathrm{m} / \mathrm{s}$ for forward motion, and $2810 \mu \mathrm{m} / \mathrm{s}$ for backward motion. Fig. 20 shows the 39 forward velocity measurements of the same device in atmospheric pressure. The resulting average forward 


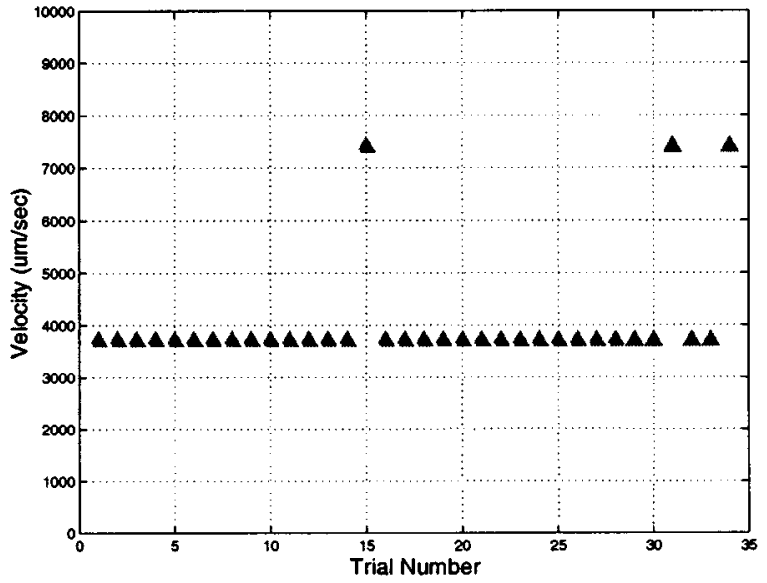

Fig. 18. Measured velocities of the forward motions in 10-mtorr pressure (average: $4024 \mu \mathrm{m} / \mathrm{s}$ )

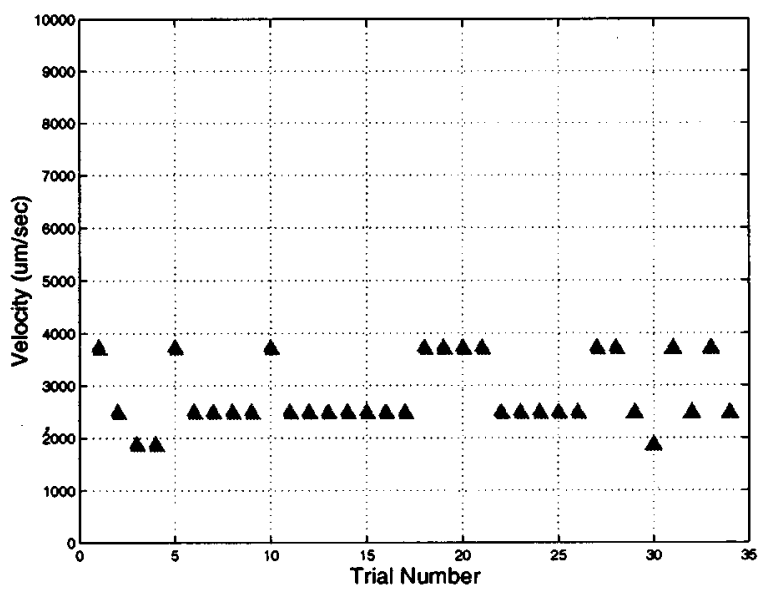

Fig. 19. Measured velocities of the backward motions in 10-mtorr pressure (average: $2810 \mu \mathrm{m} / \mathrm{s}$ ).

velocity is $3958 \mu \mathrm{m} / \mathrm{s}$, which is only marginally different from the values in 10 -mtorr pressure. This indicates that the effect of the acoustic streaming force of the air to the slider motion is minimal, despite the high-frequency horizontal oscillation of the substrate.

In order to obtain the insight on the effect of the frictional forces on the slider velocities, a number of simulations are performed with the various values of $F_{d}$ and $F_{s}$. These simulation results reveal that the slider velocity is fairly sensitive to the values of dynamic frictional force $F_{d}$, whereas the values of static frictional force $F_{s}$ has almost no effects on the slider velocity for a significantly wide range $\left(10^{-2}-10^{4} \mu \mathrm{N}\right)$. Therefore, it is concluded that the value $F_{s}=20 \mu \mathrm{N}$ reported in [4] for the slider with virtually identical size and manufacturing process is sufficient for the purpose of the performance prediction.

On the other hand, the simulation results indicate that the value of dynamic frictional force $F_{d}$ has fair effects on the simulated slider velocity. Therefore, for each of the above velocity measurements the value $F_{d}$ in (7) is adjusted to fit the simulated

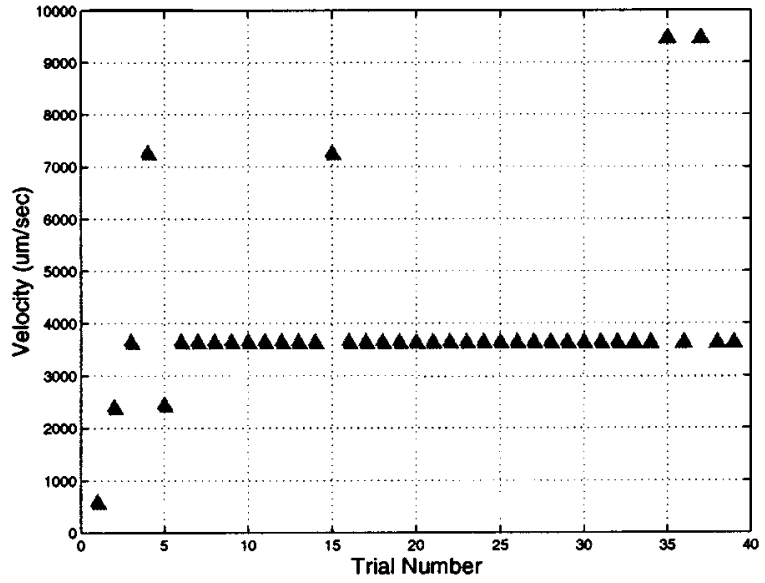

Fig. 20. Measured velocities of the forward motions in atmospheric pressure (average: $3958 \mu \mathrm{m} / \mathrm{s}$ )

slider velocity (with $F_{s}=20 \mu \mathrm{N}$ ) to the measured velocity. In order to account for unknown manufacturing error, the values of spring constant $k$ in (1) is obtained from the natural frequency of the actual device, rather than using (4). The resulting values of $F_{d}$ are $6.8 \mu \mathrm{N}$ for forward motion and $0.75 \mu \mathrm{N}$ for backward motion. The value for the forward motion is comparable to $F_{d}=5 \mu \mathrm{N}$ reported in [4] for the slider with virtually identical size and manufacturing process. The very low $F_{d}$ value in backward motion can be explained as a result of undesired "assist" of forward impacters during the backward motion-due to error in manufacturing processes, the forward impacters can be slightly resonated with backward resonance frequency $\nu_{b}$ to barely stick the slider sidewall and pull it periodically. Statistical consideration of manufacturing errors in the dynamic model would help to reduce such undesired coupling of forward and backward resonance.

\section{Discussion AND Future Work}

This paper presented a new method for on-substrate fine positioning of microscale/mesoscale discrete components [1], where component positions are finely adjusted using microlinear sliders and fixtures on the substrate. Each microlinear slider is actuated by vibratory impacts exerted by two pairs of microcantilever impacters. These microcantilever impacters are selectively resonated by shaking the entire substrate with a piezoelectric vibrator, requiring no need for built-in driving mechanisms such as electrostatic comb actuators, as reported previously [4], [5]. This selective resonance of the microcantilever impacters via an external vibration energy field [6] provides with a very simple means of controlling forward and backward motions of the microlinear slider, facilitating assembly and disassembly of a microcomponent on a substrate.

As discussed in Section I, gross positioning of a microcomponent needs to be done prior to on-substrate fine positioning using an externally resonated linear microvibromotor. Although the gross positioning could be done sequentially in pick-and-place fashion, vibratory palletization [32], a part orienting method common to centimeter-scale mechanical parts, could provide 
very efficient means of parallel gross positioning of microcomponents. During the palletization, surface adhesion forces can be virtually eliminated by applying vertical vibration in ultrasonic range, as reported in [16].

After such parallel gross positioning, an array of linear vibromotors on the substrate can be simultaneously or selectively activated to fine-position the components with an appropriate sum of input signals with different frequencies. Also, the positioned components can be selectively released later in the same manner. Designing such vibromotor array would require a careful assignment of driving frequencies to each vibromotor to avoid undesired mutual interferences. Since it is observed that the lumped parameter model presented in this paper can be off by $30 \%-40 \%$ from the experimental value in worst cases, more detailed model (perhaps finite-element models) coupled with statistical consideration of manufacturing errors would be a key to the development of such a design method.

The current fine positioning scheme, however, lacks a positive fastening means to secure the attachment of the component to the substrate that can be detached nondestructively when needed. Therefore, the design modification of the linear slider, the etched cavity, and/or anchored fixture should be investigated in order to achieve selective fastening and release of a component. Removable micromechanical latching fasteners, or micro"mouse traps," [33], could provide a possible fastening means.

One of the most promising applications of the microassembly/disassembly as described in this paper is bare-chip interconnection in multichip module (MCM), which requires a precision assembly/disassembly of mesoscale components with high-density electrical interconnection. Although the chips currently used in MCM's are typically in 5-10-mm scale, the advent of the assembly/disassembly method by using the externally resonated linear microvibromotor presented in this paper would stimulate further disintegration of subsystem components to improve the overall system modularity, which, in turn, would reduce the sizes of the components to be assembled.

\section{ACKNOWLEDGMENT}

The authors would like to thank the staff of the Solid-State Electronics Laboratory, The University of Michigan at Ann Arbor, for their assistance.

\section{REFERENCES}

[1] K. Saitou and S. J. Wou, "Externally-resonated linear micro vibromotors for micro assembly," in Proc. SPIE. Microrobot. Micromanipulation Conf, vol. 3519, Boston, MA, Nov. 1998, pp. 128-139.

[2] K. Saitou, S. J. Wou, and D.-A. Wang, "Assembly and disassembly of bare chips using on-substrate linear microvibromotor arrays," in Proc. EcoDesign'99, Tokyo, Japan, pp. 818-823.

[3] S. J. Wou, "Externally resonated linear microvibromotor for micro assembly," M.S. thesis, Dept. Mech. Eng., Univ. Michigan at Ann Arbor, Ann Arbor, MI, 1998.

[4] M. J. Daneman, N. C. Tien, O. Solgaard, A. P. Pisano, K. Y. Lau, and R. S. Muller, "Linear microvibromotor for positioning optical components,” J. Microelectromech. Syst., vol. 5, pp. 159-165, Sept. 1996.
[5] A. P. Lee and A. P. Pisano, "Polysilicon angular microvibromotors," J. Microelectromech. Syst., vol. 1, pp. 70-76, June 1992.

[6] T. Yasuda, I. Shimoyama, and H. Miura, "Microrobot actuated by a vibration energy field," Sens. Actuators A, Phys., vol. 43, pp. 366-370, 1994.

[7] C. G. Keller and R. T. Howe, "Hexsil tweezers for teleoperated microassembly," J. Microelectromech. Syst., vol. 5, pp. 72-77, Jan. 1997.

[8] R. S. Fearing, "Survey of sticking effects for micro parts handling," in Proc. Int. Intell. Robot. Syst., vol. 2, 1995, pp. 212-217.

[9] L. Saggere, S. Kota, and S. B. Crary, "A new design for suspension systems of linear microactiators," in ASME Proc. Int. Mech. Eng. Congr. Expo., vol. DSC 55-2, 1994, pp. 671-675.

[10] H. J. Yeh and J. S. Smith, "Fluidic self-assembly of GaAs microstructures on Si substrates," Sens. Mater. vol. 6, no. 6, pp. 319-332, 1994.

[11] M. A. Hadley, "Self-orienting fluidic transport (SOFT) assembly of liquid crystal displays," presented at the Defence Manufact. Conf., Palm Springs, CA, Dec. 1997.

[12] A. Terfort, N. Bowden, and G. M. Whitesides, "Three-dimensional selfassembly of millimeter-scale components," Nature, vol. 386, no. 6621, p. 162, Mar. 1997.

[13] A. Terfort and G. M. Whitesides, "Self-assembly of an operating electrical circuit based on shape complementarity and the hydrophibic effects," Adv. Mater, vol. 10, no. 6, pp. 470-473, 1998.

[14] K. Hosokawa, I. Shimoyama, and H. Miura, "Two-dimensional microself-assembly using the surface tension of water," Sens. Actuators A Phys., vol. 57, no. 2, pp. 117-125, Nov. 1995.

[15] M. B. Cohn, C.-J. Kim, and A. P. Pisano, "Self-assembling electrical networks: An application of micromachining technology," in IEEE Int. Solid-State Sens. Actuators Conf., San Francisco, CA, 1991, pp. 490-493.

[16] K. Böhringer, K. Goldberg, M. Cohn, R. Howe, and A. Pisano, "Parallel microassembly with electrostatic force fields," in Proc. IEEE Int. Robot. Automat. Conf., 1997, pp. 1204-1211.

[17] M. W. Judy, Y.-H. Cho, R. T. Howe, and A. P. Pisano, "Self-adjusting microstructures (SAMS)," in Proc. IEEE Microelectromech. Syst., 1991, pp. 51-56.

[18] S. R. Burgett, K. S. J. Pister, and R. S. Fearing, "Three dimensional structures made with microfabricated hinges," in ASME Int. Mech. Eng. Congr. Expo., 1992, pp. 1-11.

[19] R. Prasad, K.-F. Böhringer, and N. C. MacDonald, "Design, fabrication, and characterization of single crystal silicon latching snap fasteners for micro assembly," in ASME Int. Mech. Eng. Congr. Expo., 1995, pp. 917-923.

[20] M. B. Cohn, K. F. Böhringer, J. M. Noworolski, A. Singh, C. G. Keller, K. Y. Goldberg, and R. T. Howe, "Microassembly technologies for MEMS," in Proc. SPIE Microfluid. Devices Conf., vol. 3515, Santa Clara, CA, Sept. 1998, pp. 2-16.

[21] A. P. Lee and A. P. Pisano, "Repetitive impact testing of micro mechanical structures," in Proc. ASME Winter Annu. Meeting, Dallas, TX, 1990, pp. 51-67.

[22] Y.-H. Cho, A. P. Pisano, and R. T. Howe, "Viscous damping model for laterally oscillating microstructures," J. Microelectromech. Syst., vol. 3, pp. 81-87, June 1994.

[23] W. N. Sharpe, Jr., B. Yuan, and R. Vaidyanathan, "Measurements of Young's modulus, Possisson's ratio, and tensile strength of polysilicon," in Proc. IEEE Microelectromech. Syst., 1996, pp. 424-429.

[24] S. W. Shaw and P. J. Holmes, "A periodically forced piecewise linear oscillator," J. Sound and Vibration, vol. 90, no. 1, pp. 129-155, 1983.

[25] S. W. Shaw, "Forced vibrations of a beam with one-sided amplitude constraint: Theory and experiment," J. Sound and Vibration, pp. 199-211, 1985.

[26] M. S. Heiman, P. J. Sherman, and A. K. Bajaj, "On the dynamics and stability of an inclined impact pair," J. Sound and Vibration, vol. 114, no. 3, pp. 535-547, 1987

[27] T. O. Dalrymple, "Numerical solutions to vibroimpact via an initial value problem formulation," J. Sound and Vibration, vol. 132, no. 1, pp. 19-32, 1989.

[28] S. H. Strogatz, Nonlinear Dynamics and Chaos: With Applications to Physics, Biology, Chemistry, and Engineering. Reading, MA: Addison-Wesley, 1994

[29] P. Y. Papalambros and D. J. Wilde, Principles of Optimal Design. Cambridge, U.K.: Cambridge Univ. Press, 1988.

[30] S. Kirkpatrick, C. D. Gellat, and M. P. Vecchi, "Optimization by simulated annealing," Science, vol. 220, pp. 671-680, 1983.

[31] R. E. Walpole and R. H. Myers, Probability and Statistics for Engineers and Scientists. New York: Macmillan, 1985. 
[32] P. H. Moncevicz, M. J. Jakiela, and K. T. Ulrich, "Orientation and insertion of randomly presented parts using vibratory agitation," in Proc. ASME 3rd Flexible Assembly Syst. Conf., vol. DE-33, A. H. Soni, Ed., New York, NY, Sept. 1991, pp. 41-47.

[33] K. Saitou and M. J. Jakiela, "Design of a self-closing compliant 'mouse trap' for micro assembly," in ASME Int. Mech. Eng. Congr. Expo., vol. DSC-59, 1996, pp. 421-426.

Kazuhiro Saitou received the $\mathrm{Ph}$.D. degree in mechanical engineering from the Massachusetts Institute of Technology, Cambridge, in 1996.

He is currently an Assistant Professor in the Department of Mechanical Engineering and Applied Mechanics, The University of Michigan at Ann Arbor. His research interests include design optimization, design for assembly, microassembly, and evolutionary computation in design.
Dung-An Wang received the M.S. degree in mechanical engineering from the National Taiwan University, Taiwan, R.O.C., in 1996, and is currently working toward the Ph.D. degree in the mechanical engineering and applied mechanics at The University of Michigan at Ann Arbor.

His research interests are microassembly and design of dwell mechanism.

Soungiin J. Wou received the B.S. degree in mechanical engineering from the University of Minnesota, Twin Cities, in 1996, and the M.S.E. degree in mechanical engineering from The University of Michigan at Ann Arbor, in 1998.

$\mathrm{He}$ is currently a Research Engineer with Bishop Steering Technology, Sydney, Australia. 